\title{
STRICTLY BARRELLED DISKS IN INDUCTIVE LIMITS OF QUASI-(LB)-SPACES
}

\author{
CARLOS BOSCH \\ Department of Mathematics \\ I.T.A.M. \\ Río Hondo \#1, Col. Tizapán San Angel \\ México 01000, D.F., Mexico \\ THOMAS E. GILSDORF \\ Department of Mathematics \\ University of North Dakota \\ Grand Forks, ND 58202-8376, USA
}

(Received June 9, 1995)

\begin{abstract}
A strictly barrelled disk $B$ in a Hausdorff locally convex space $E$ is a disk such that the linear span of $B$ with the topology of the Minkowski functional of $B$ is a strictly barrelled space. Valdivia's closed graph theorems are used to show that closed strictly barrelled disk in a quasi-(LB)-space is bounded. It is shown that a locally strictly barrelled quasi-(LB)-space is locally complete. Also, we show that a regular inductive limit of quasi-(LB)spaces is locally complete if and only if each closed bounded disk is a strictly barrelled disk in one of the constituents.
\end{abstract}

KEY WORDS AND PHRASES. Quasi-(LB)-space, strictly barrelled space, inductive limit.

1991 AMS SUBJECT CLASSIFICATION CODE. Primary 46A13, 46A08. Secondary $46 \mathrm{~A} 30$.

1. INTRODUCTION.

Throughout this paper, we use the word space to denote a Hausdorff locally convex space. An absolutely convex set will be called a disk. If $A$ is a disk in a space $E$, its linear span $E_{A}$ may be endowed with the semi-normed topology 
given by the Minkowski functional of $A$. When distinction is needed, we will denote this topology by $r_{A}$. When $A$ is a bounded disk, it is easy to see that $E_{A}$ is normed and that $i d: E_{A} \rightarrow E$ is continuous. If $E_{A}$ is a Banach space (resp. Baire space), we call $A$ a Banach (resp. Baire) disk. If every bounded subset of $E$ is contained in a bounded Banach (resp. Baire) disk, we say that $E$ is locally complete (resp. locally Baire). Locally complete spaces are also called fast complete, and according to [1;5.1.6, pg. 152], a space is locally complete if and only if every closed bounded disk is already a Banach disk.

DEFINITION 1.1: Following [2], a space $E$ is strictly barrelled if given any ordered absolutely convex web $\mathcal{W}$ on $E$ there exists a strand $(W(k))=\{W(k): k \in$ $\boldsymbol{N}$ \} of $\mathcal{W}$ such that for each positive integer $k$, the closure $\overline{W(k)}$ is a zero neighborhood in $E$, where $W(k)$ denotes the $k$ th member of a strand $(W(k))$.

DEFINITION 1.2: Let $A$ be a disk. If $E_{A}$ is a strictly barrelled space, we will say that $A$ is a strictly barrelled disk. If every bounded set is contained in a strictly barrelled disk, we say that $E$ is locally strictly barrelled.

REMARK 1.3: Using [1; chapt. 9] and [2; Prop. 6.17, pg. 160],

locally complete $\Rightarrow$ locally Baire $\Rightarrow$ locally strictly barrelled.

These implications cannot be reversed; the first by $[1 ; 1.2 .12 \mathrm{pg} .17]$, the second by [2; Prop. 17, pg. 160 \& Note 4, pg. 162]. Valdivia defines quasi-(LB)-spaces in [2], and proves a webbed-space equivalence in [2; Th. 4.1, pg. 153]. We will use this equivalence as our definition below.

DEFINITION 1.4: A space with an ordered, absolutely convex strict web is called a quasi-(LB)-space.

\section{QUASI-(LB)-SPACES AND STRICTLY BARRELLED DISKS.}

The following generalizes [3; Th. 3, pg. 173] and [4; Th. 1, pg. 222].

THEOREM 2.1: Let $B$ be a closed strictly barrelled disk in a quasi- (LB)-space. Then $B$ is bounded.

PROOF: Let $(E, \tau)$ be the quasi- (LB)-space that contains $B$. Denote by $\eta$ the topology induced on $E_{B}$ by the following system of neighborhoods: $\left\{\left(n^{-1} B\right) \cap V: V\right.$ is a $\tau$-closed zero neighborhood, $n \in N\}$. Using the ordered strict web on $(E, \tau)$ and the 
construction in [4; Th. 1, pg. 222], we have that $\left(E_{B}, \eta\right)$ is a quasi-(LB)-space. The map id: $\left(E_{B}, \eta\right) \rightarrow\left(E_{B}, p_{B}\right)$ is continuous and $\left(E_{B}, p_{B}\right)$ is strictly barrelled. Therefore, by [2; Th. 6.5(a), pg. 163], this map is open, implying that for any $\tau$ zero neighborhood $V, \quad i d(B \cap V)$ is a neighborhood of zero in $\left(E_{B} \cdot r_{B}\right)$. In particular, there exists $a>0$ such that $a B \subset B \cap V \subset V$. We conclude that $B$ is $T$ bounded.

The result that follows uses the closed graph theorem of Valdivia [2].

THEOREM 2.2: Any locally strictly barrelled quasi-(LB)-space is locally complete.

PROOF: Assume $(E, t)$ is such a space and suppose $A$ is bounded in $E$. There is a , bounded disk $B \supset A$ such that $\left(E_{B}, p_{B}\right)$ is strictly barrelled. Because $i d:\left(E_{B}, p_{B}\right)$ $\rightarrow\left(E_{B}, t\right)$ is continuous, [2; Th. $7.6 \mathrm{pg}$. 164] shows that there is a Fréchet space $F$ for which $E_{B}=i d\left(E_{B}\right) \subset F$ and the following injections are continuous: $\left(E_{B}, P_{B}\right) \rightarrow$ $F \rightarrow\left(E_{B}, t\right)$. Hence, there is a bounded Banach disk $D$ in $F$, with $A \subset B \subset D$, and $D$ is a bounded Banach disk in $E$ as well.

\section{INDUCTIVE LIMITS.}

In this section we consider sequences $\left(E_{n}, t_{n}\right), n \in \mathbf{N}$ of spaces with $E_{l} \subset E_{2} \subset \ldots$, and for every positive integer $n, E_{n}$ injects continuously into $E_{n+l}$. We put $E=$ ind $_{n} E_{n}$ for the inductive limit. Recall that an inductive limit is called regular if for any of its bounded subsets, there is a constituent space such that the subset is contained in and bounded in that constituent.

THEOREM 3.1: Let $E=$ ind $_{n} E_{n}$ be an inductive limit of quasi-(LB)-spaces. Suppose $B$ is a disk in $\left(E_{n}, t_{n}\right)$. Then:

(a) If there exists $m \geq n$ such that $B$ is a closed strictly barrelled disk in $\left(E_{m}\right.$, $\left.t_{m}\right)$, then $B$ is a closed bounded strictly barrelled disk in both $\left(E_{n}, t_{n}\right)$ and $\left(E_{m}, t_{m}\right)$. Moreover, $B$ is contained in a bounded Banach disk in $\left(E_{n}, t_{n}\right)$ and $\left(E_{m}, t_{m}\right)$.

(b) If (a) holds for every bounded disk in $E_{n}$, then $E_{n}$ is locally complete. 
(c) If $E$ is regular and locally complete, then $E_{n}$ is locally complete for every positive integer $n$.

PROOF: $(a)$ : If the assumptions are satisfied, then from the continuity of $i d:\left(E_{n}\right.$, $\left.t_{n}\right) \rightarrow\left(E_{m}, t_{m}\right), B$ is $t_{n}$ - closed. As a strictly barrelled, closed disk in $\left(E_{m}, t_{m}\right), B$ is $t_{n}$ - bounded by Theorem 2.1. We use Theorem 2.2 in both $\left(E_{m}, t_{m}\right)$ and $\left(E_{n}, t_{n}\right)$ to conclude that $B$ is contained in a bounded Banach disk in both spaces.

(b): Obvious consequence of $(a)$.

(c): Let $E$ be any fixed natural number and let $A \subset E_{n}$ be bounded. By the assumptions and topology on $E, A$ is bounded in $E$, and contained in an $E$ - closed, bounded Banach disk $D$, where $D$ itself is contained in and bounded in some $\left(E_{m}\right.$, $\left.t_{m}\right)$; clearly $m \geq n$. As $i d:\left(E_{m}, t_{m}\right) \rightarrow E$ is continuous, $D$ is $t_{m}$ - closed and of course is a bounded Banach disk there. We apply part (a) to the disk $D \cap E_{n}$ and we are done.

In [5] we have that if each $\left(E_{n}, t_{n}\right)$ is webbed and locally complete, then is $E=$ $i_{n} E_{n}$ regular if and only if it is locally complete. One can ask what happens if the inductive limit is regular but the spaces $\left(E_{n}, t_{n}\right)$ are not locally complete; see for example [6] and [7]. It is not difficult to prove a similar type of result using quasi-(LB)-spaces; the details follow. Compare also [4; Th. 3, pg 223] and [3; Th.5, pg. 174].

THEOREM 3.2: Suppose each $\left(E_{n}, t_{n}\right)$ is a quasi-(LB)-space and $E=$ ind $_{n} E_{n}$ is regular. Then $E$ is locally complete if and only if for each closed, bounded disk $B \subset E_{n}$, there is an $m \in N$ such that $B$ is a strictly barrelled disk in $\left(E_{m}, t_{m}\right)$.

PROOF: If $E$ is locally complete, the conclusion follows directly from from 3.1 (c). Conversely, take a closed, bounded disk $B$ in $E$. There is an $n \in \mathbf{N}$ such that $B$ $\subset E_{n}$ and is $t_{n}$-bounded, and there is an $m \in \mathbf{N}$ with $B \subset E_{m}$ and $B$ is a strictly barrelled disk. If $m>n$, we use 3.1 (a). On the other hand, if $n \geq m$, then 2.1 tells us that $n=m$ and $(a)$ of 3.1 applies. In either case, $E$ is locally complete.

We want to construct a regular inductive limit of non-locally complete quasi(LB)-spaces, but first we need:

LEMMA 3.3: A finite product of locally convex spaces is locally complete if and only if each space is locally complete. 
PROOF: One may use bornologies, [8; 3.2(3), pg 43], to prove that any product of locally complete spaces is locally complete. Conversely, let $E=F \times G$, and assume that $E$ is locally complete. Suppose, without loss of generality, that $F$ is not locally complete. This means there is a disk $B$, closed and bounded in $F$, and $B$ is not a Banach disk in $F$. Then $B^{\prime}=B \times\{0\}$ is an $E$ - closed and bounded disk that is not a Banach disk, a contradiction. Hence, $F$ is locally complete. The proof for general finite products can is done by induction.

EXAMPLE 3.4: Let $E_{0}$ be an non-regular (LB)-space. Then $E_{0}$ is a quasi-(LB)space by [2; Prop 3.5, pg 152]. For each positive integer $n$, put $E_{n}=\oplus\left\{E_{0}: i=1,2, \ldots\right\} \cong \Pi\left\{E_{O}: i=1,2, \ldots\right\}$. The lemma, the non-regularity of $E_{0}$ and [2; Prop 3.3, pg 151] imply that each $E_{n}$ is a non-locally complete quasi(LB)-space.

Set $E=i n d_{n} E_{n}=\oplus\left\{E_{O}: n \in \mathbf{N}\right\}$. As a direct sum, if $A \subset E$ is bounded, then there is a finite subset $I$ of $\mathbf{N}$ such that $A$ is bounded in $\oplus\left\{E_{O}: i \in I\right\}$. If $\left.n=\max _{\{} i: i \in I\right\}$, then $A$ is bounded in $E_{n}$, and $E$ is therefore regular. Next, we use 3.2. Let $B \subset E_{I}=E_{0}$ be a closed, bounded disk that is not a Banach disk. Using the defintion of the direct sum topology $t$ of $E$ and the fact that $t$ induces on $E_{O}$ its own topology, we have that $B$ is a closed bounded disk in $E$, also. The disk $B$ cannot be a Banach disk in $E$, so $E$ is not locally complete. From 3.2, we see that $B$ is in fact a really bad disk; not only is it a non-Banach disk in $E$, it cannot be a strictly barrelled disk in any $E_{n}$.

ACKNOWLEDGEMENT. Research for the second author was supported as part of a Solomon Lefshetz Fellowship at el Centro de Investigaciones y Estudios Avanzados, Mexico City, Mexico, 1992-1993. 


\section{REFERENCES}

[1] PEREZ-CARRERAS, P., BONET, J.; Barrelled Locally Convex Spaces, North Holland Math. Studies, 131 , (1987).

[2] VALDIVIA, M.; Quasi-(LB)-spaces, J. London Math. Soc., (2) 35, (1987), pp. 149-168.

[3] QIU, J. H.; Dieudonné-Schwartz theorem in inductive limits of metrizable spaces II, Proc. AMS, 108, no. 1, Jan. (1990), pp. 171-175.

[4] BOSCH, C., KUCERA, J.; Bounded sets in inductive limits of $\mathcal{X}$-spaces, $\underline{\text { Czech }}$ J. Math, 43 (118), (1993), pp. 221-223.

[5] BOSCH, C., KUCERA, J.; Bounded sets in fast complete inductive limits, Int. J. Math. \& Math. Sci., 7, no. 3, (1984), pp. 615-617.

[6] GILSDORF, T.; Local Baire-like properties and inductive limits, preprint.

[7] GILSDORF, T.; Regular inductive limits of $\mathcal{X}$-spaces, Collect. Math., 42, no. 1 , 1991 (1992), pp. 45-49.

[8] HOGBE-NLEND, H.; Bornologies and Functional Analysis, North Holland Math. Studies, 26, (1977). 


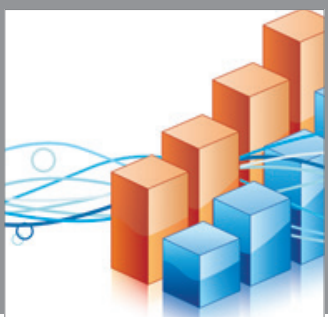

Advances in

Operations Research

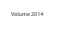

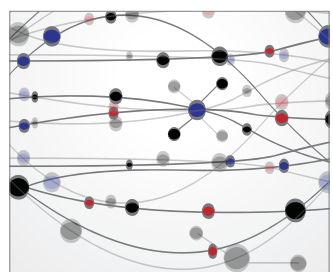

\section{The Scientific} World Journal
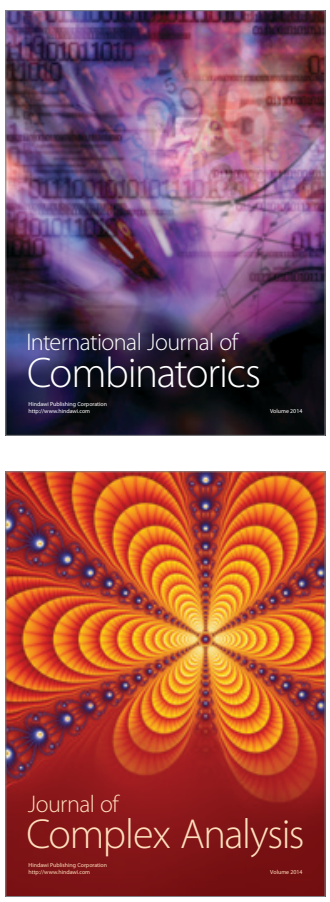

International Journal of

Mathematics and

Mathematical

Sciences
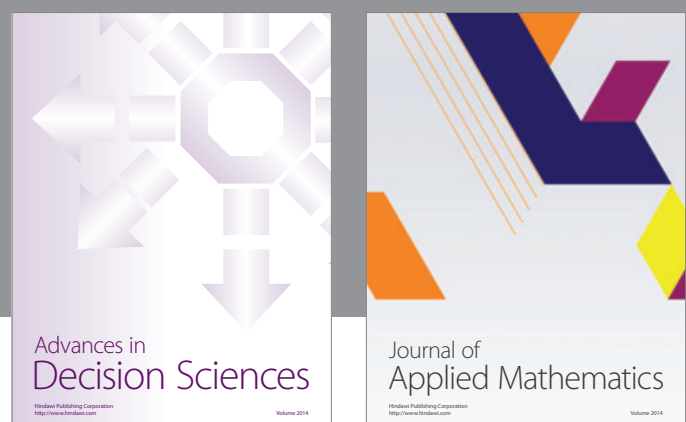

Journal of

Applied Mathematics
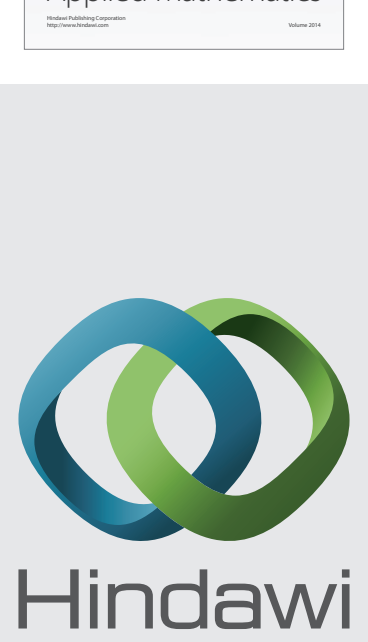

Submit your manuscripts at http://www.hindawi.com
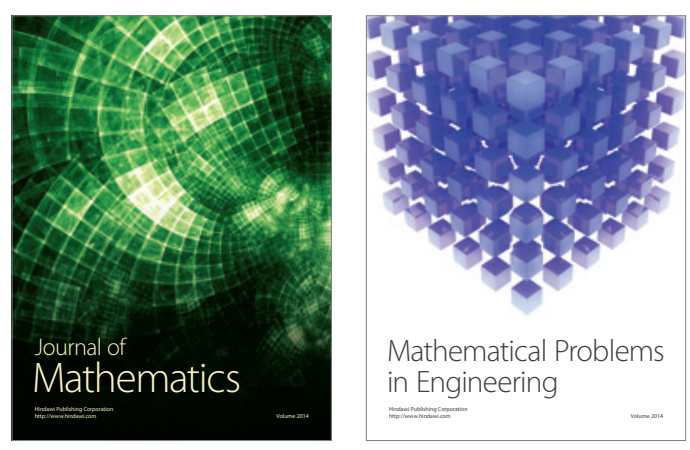

Mathematical Problems in Engineering
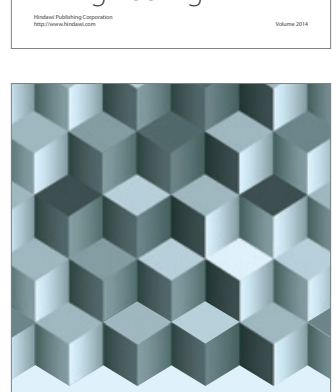

Journal of

Function Spaces
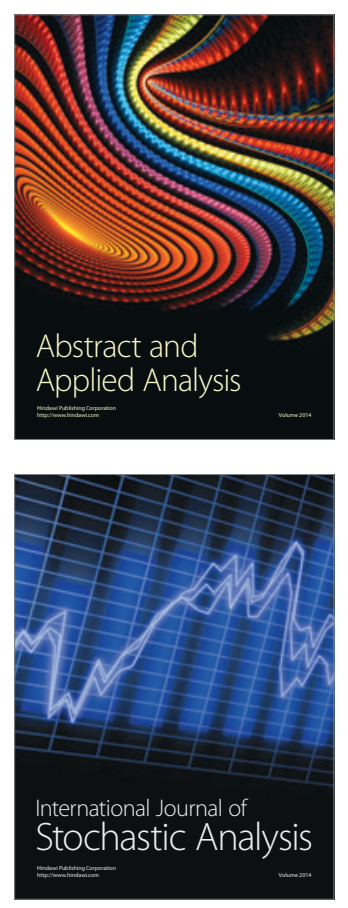

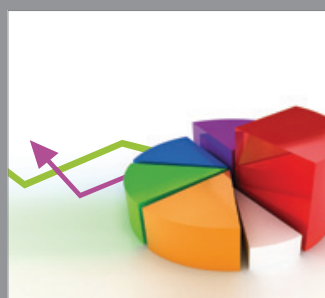

ournal of

Probability and Statistics

Promensencen
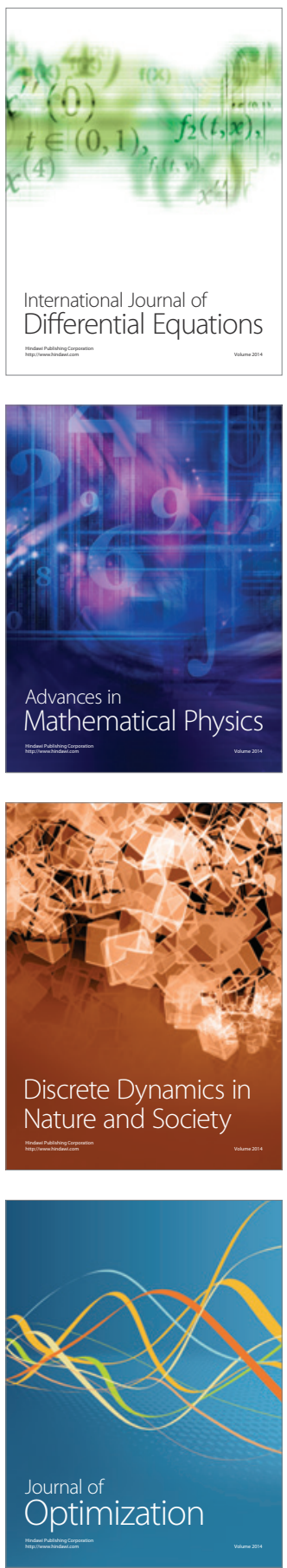